\title{
Impact of concurrent amiodarone treatment on the tolerability and efficacy of carvedilol in patients with chronic heart failure
}

\author{
P S Macdonald, A M Keogh, C Aboyoun, M Lund, R Amor, D McCaffrey
}

\begin{abstract}
Objective-To assess the safety and efficacy of carvedilol when administered to heart failure patients already receiving amiodarone.

Design-Retrospective analysis of the clinical outcome of 230 patients treated with carvedilol for chronic heart failure, stratified according to whether they were already receiving amiodarone (amiodarone group, 80 patients) or not (nonamiodarone group, 130 patients) at baseline.

Setting-Heart failure clinic at a university affiliated public teaching hospital.

Main outcome measures-Incidence of adverse events; changes in functional status and echocardiographic dimensions at three months.

Results-Adverse reactions to carvedilol occurred in $33(41 \%)$ of the amiodarone group and $43(29 \%)$ of the non-amiodarone group ( $p=0.049)$. Carvedilol was discontinued in $21(26 \%)$ of the amiodarone group and $37(25 \%)$ of the non-amiodarone group (NS). The clinical outcome at three months did not differ significantly between the two groups; $31(39 \%)$ of the amiodarone group improved their New York Heart Association status, 28 (35\%) were unchanged, and $21(26 \%)$ deteriorated compared with 67 $(45 \%), 51(34 \%)$, and $32(21 \%)$, respectively, for the non-amiodarone group (NS). Both groups had highly significant decreases in heart rate and left ventricular end systolic dimension, and a significant increase in left ventricular ejection fraction after three months of carvedilol treatment, with no significant differences between the groups. Conclusions-The beneficial effects of carvedilol on left ventricular remodelling, systolic function, and symptomatic status are not affected by concurrent treatment with amiodarone. Adverse reactions necessitating cessation of carvedilol are no more frequent in patients receiving amiodarone. (Heart 1999;82:589-593)
\end{abstract}

Keywords: carvedilol; amiodarone; heart failure

Carvedilol, a non-selective $\beta$ adrenergic blocking drug with $\alpha$ blocking and antioxidant properties, has been shown to produce a number of benefits in selected patients with chronic heart failure. These benefits include reverse remodelling of the dilated left ventricle, ${ }^{1}$ improved left ventricular systolic function, ${ }^{2-4}$ improved symptomatic status, ${ }^{2}{ }^{35}$ reduced progression of heart failure, ${ }^{6}$ reduced hospitalisation, and reduced mortality. ${ }^{57}$ Amiodarone has also been shown to produce beneficial effects in heart failure including improved left ventricular systolic function, ${ }^{8-10}$ improved symptoms, ${ }^{10}$ and possibly improved survival. ${ }^{11}{ }^{12}$ Amiodarone, like carvedilol, has antiadrenergic properties although these are mediated via different pharmacological actions. These actions include non-competitive blockade of $\beta$ and $\alpha$ adrenergic receptors, ${ }^{13}{ }^{14}$ possibly mediated via down regulation of receptor protein, ${ }^{15}$ and inhibition of vesicular monoamine transport into presynaptic sympathetic neurons. ${ }^{16}$ The extent to which these properties contribute to amiodarone's clinical effects is unclear.

The safety and efficacy of amiodarone in patients with heart failure, as shown in randomised controlled trials, has led to a progressive increase in its use in this context. ${ }^{17}$ Therefore, it is likely that many patients being considered for carvedilol treatment will already be receiving amiodarone. The aim of this study was to assess the tolerability and efficacy of carvedilol in chronic heart failure patients who were already being treated with amiodarone.

\section{Methods}

PATIENT POPULATION

We retrospectively analysed the outcomes of 230 patients who received carvedilol for the treatment of chronic heart failure. All patients included in this analysis were attending a heart failure and heart transplant assessment clinic at our institution and had been receiving heart failure treatment for at least three months before starting carvedilol treatment. All patients had left ventricular systolic dysfunction at baseline as defined by a left ventricular fractional shortening $<28 \%$ on echocardiography. Patients were not considered for carvedilol if they had any of the following at baseline assessment: cardiogenic shock; intractable pulmonary or systemic oedema; heart failure requiring intravenous inotropic or mechanical support; bradycardia with heart rate $<50$ beats/min; systemic hypotension with blood pressure $<80 / 50 \mathrm{~mm} \mathrm{Hg}$.

\section{CARVEDILOL ADMINISTRATION}

Throughout the duration of this study, carvedilol was available as an investigational agent for the treatment of heart failure (special access scheme of the therapeutic and goods administration division of the Australian Department of Health). Its use for this indication was approved by the St Vincent's Hospital human ethics and research committee. The 
Table 1 Selected baseline characteristics

\begin{tabular}{|c|c|c|c|}
\hline & $\begin{array}{l}\text { Amiodarone } \\
(n=80)\end{array}$ & $\begin{array}{l}\text { No amiodarone } \\
(n=150)\end{array}$ & p Value \\
\hline Age (years) & $56(1)$ & $53(1)$ & NS \\
\hline Sex (\% male $)$ & 96 & 80 & $<0.001$ \\
\hline Diagnosis (cardiomyopathy:IHD:other) & $43: 32: 5$ & $84: 54: 12$ & NS \\
\hline Duration (months) & $27(4)$ & $33(4)$ & NS \\
\hline \multicolumn{4}{|l|}{ NYHA class } \\
\hline I & 2 & 8 & NS \\
\hline II & 14 & 31 & NS \\
\hline III & 44 & 68 & NS \\
\hline IV & 20 & 43 & NS \\
\hline Heart rate (beats/min) & $82(1)$ & $85(1)$ & NS \\
\hline \multicolumn{4}{|l|}{ Rhythm } \\
\hline Sinus & $50(63 \%)$ & $126(84 \%)$ & \\
\hline Atrial fibrillation & $27(34 \%)$ & $15(10 \%)$ & $<0.0001$ \\
\hline Other & $3(3 \%)$ & $9(6 \%)$ & \\
\hline \multicolumn{4}{|l|}{ Echocardiographic measurements } \\
\hline LVEDD $(\mathrm{mm})$ & $74(1)$ & $73(1)$ & NS \\
\hline $\operatorname{LVESD}(\mathrm{mm})$ & $65(1)$ & $63(1)$ & NS \\
\hline Fractional shortening (\%) & $13(1)$ & $14(1)$ & NS \\
\hline Ejection fraction $(\%)$ & $23(1)$ & $24(1)$ & NS \\
\hline 6 minute walk $(\mathrm{m})$ & $408(15)$ & $429(11)$ & NS \\
\hline \multicolumn{4}{|l|}{ Treatment $(\%)$} \\
\hline ACE inhibitors & 100 & 96 & NS \\
\hline Diuretics & 98 & 89 & 0.02 \\
\hline Digoxin & 78 & 76 & NS \\
\hline Vasodilators & 58 & 48 & NS \\
\hline Antithrombotic & 88 & 73 & $<0.01$ \\
\hline
\end{tabular}

ACE, angiotensin converting enzyme; IHD, ischaemic heart disease; LVEDD, left ventricular end diastolic dimension; LVESD left ventricular end systolic dimension.

decision to use carvedilol was at the discretion of the attending cardiologist (PSM or AMK). Carvedilol was started at a dose of $3.125 \mathrm{mg}$ twice daily. The dose of carvedilol was doubled at two weekly intervals as tolerated up to a target dose of $25 \mathrm{mg}$ twice daily.

ASSESSMENT AND FOLLOW UP

All patients underwent clinical assessment at baseline, one month, and then every three months after starting carvedilol treatment. This included an evaluation of New York Heart Association (NYHA) functional status and physical examination including measurement of supine resting heart rate and blood pressure. Patient symptomatic outcome was graded at each follow up evaluation as improved, unchanged, or worse according to changes in NYHA status. Patients who died, underwent transplantation, or who were unable to tolerate carvedilol were considered worse. An echocardiogram and six minute walk test were performed at baseline, three months', and 12 months' follow up. Left ventricular dimensions were made using two dimensionally guided $M$ mode echocardiography according to the American Society of Echocardiography stand-

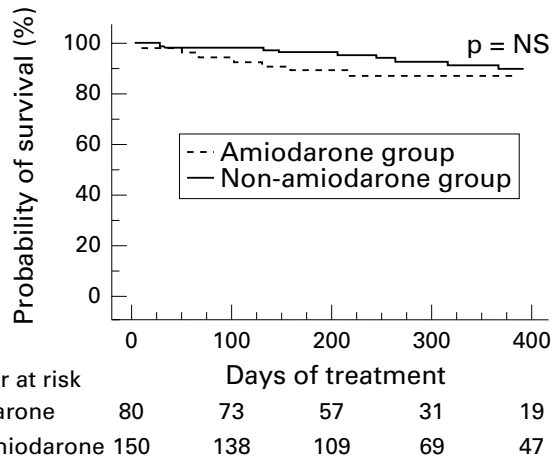

Non-amiodarone $150 \quad 138 \quad 109 \quad 69 \quad 47$

Figure 1 Kaplan-Meier cumulative survival plot from start of carvedilol treatment stratified according to whether the patients were receiving amiodarone at baseline. ards for left ventricular dimensions. Left ventricular ejection fraction was calculated from echocardiographic $M$ mode dimensions using the formula of Teichholz. ${ }^{18}$ All echocardiograms were reported by an independent cardiologist who was not involved in the follow up of the patients. This cardiologist was aware that the patients were receiving carvedilol but was unaware of which patients were also receiving amiodarone. In addition the following major clinical events were recorded: worsening heart failure resulting in hospitalisation or prolongation of hospitalisation; hospitalisation for any other reason; heart transplantation; and death.

STATISTICAL ANALYSIS

Unless otherwise stated data are presented as mean (SEM). Comparisons between the groups were made using unpaired $t$ tests for continuous variables and $\chi^{2}$ analyses for categorical variables. Kaplan-Meier cumulative survival curves were constructed for each group to compare survival and freedom from hospitalisation. Differences between KaplanMeier curves were tested for significance using the Mantel-Cox log rank test. A value of $\mathrm{p}<0.05$ was considered significant.

\section{Results}

PATIENT POPULATION

Selected baseline characteristics for the study population are shown in table 1 , stratified according to the whether or not the patients were receiving amiodarone at baseline. The two groups did not differ significantly with respect to age, cause, and duration of heart failure, NYHA class, six minute walk distance, left ventricular dimensions, or systolic function as assessed by echocardiography. Patients receiving amiodarone at baseline were more likely to be male, to be in atrial fibrillation, and to be receiving either warfarin or aspirin.

Eighty patients $(35 \%)$ were receiving amiodarone at the time they started carvedilol treatment. The indications for amiodarone were atrial tachyarrhythmias in 47 patients (most commonly atrial fibrillation) and ventricular tachyarrhythmias in 25 patients. Eight patients were receiving amiodarone on the basis of their left ventricular dysfunction alone in the absence of any documented arrhythmia. The duration of amiodarone treatment before the initiation of carvedilol varied widely from one week to seven years, with an average duration of 15 (18) months (median seven months). Twenty patients had been maintained on amiodarone for less than three months before starting carvedilol treatment. The average maintenance dose of amiodarone was 190 (41) $\mathrm{mg} /$ day. The maintenance dose of amiodarone was not altered at the time carvedilol treatment was started.

The average daily carvedilol dose after three months of treatment was 36 (2) $\mathrm{mg}$ in the amiodarone group and 36 (1) $\mathrm{mg}$ in the non-amiodarone group (NS). Overall, $52 \%$ of patients achieved the target daily dose of $50 \mathrm{mg}$ by three months. Thirty two per cent were 
Table 2 Non-fatal adverse events

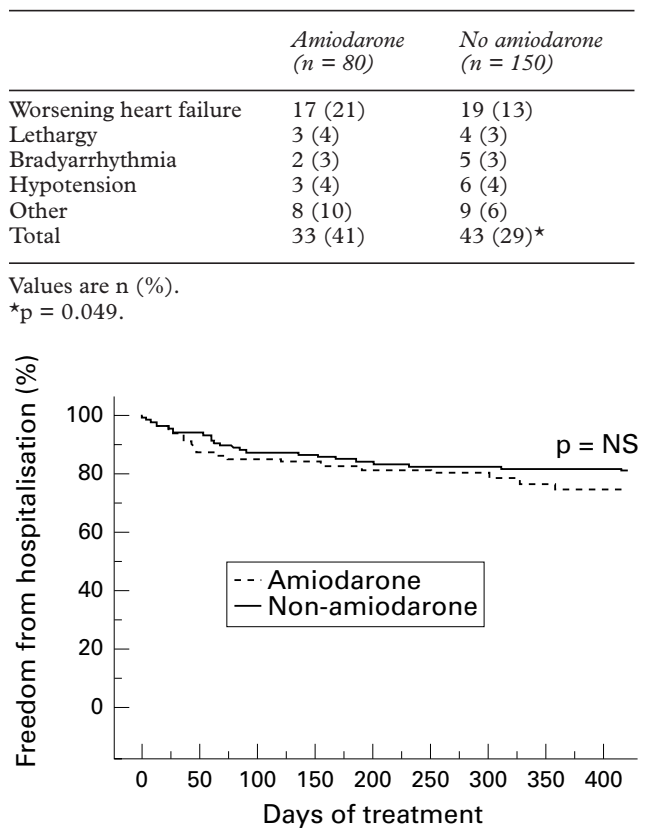

Figure 2 Kaplan-Meier cumulative plot showing freedom from hospitalisation from start of carvedilol treatment stratified according to whether or not the patients were receiving amiodarone at baseline.

maintained on $25 \mathrm{mg}$ daily and $16 \%$ on a lower dose. These percentages did not differ between the two groups.

\section{SURVIVAL AFTER STARTING CARVEDILOL TREATMENT}

A Kaplan-Meier cumulative survival plot for the two groups is shown in fig 1 . One year actuarial survival for the amiodarone group was 88 (4)\% compared with 92 (3)\% for the non-amiodarone group $(p=0.12)$. Actuarial transplant free survival for the amiodarone group was 82 (5)\% at one year compared with $84(4) \%$ for the nonamiodarone group $(\mathrm{p}=0.24)$.

TOLERABILITY OF CARVEDILOL: NON-FATAL ADVERSE EVENTS AND REHOSPITALISATION Serious adverse events excluding death or transplantation occurred in $41 \%$ of the amiodarone group compared with $29 \%$ of patients not treated with amiodarone $(\mathrm{p}=0.049)$. Non-fatal adverse events are summarised in table 2. In spite of the tendency towards more adverse events in the amiodarone group, permanent withdrawal of carvedilol was no

Table 3 Efficacy of carvedilol after three months of treatment

\begin{tabular}{llll}
\hline & Amiodarone & No amiodarone & p Value \\
\hline Symptomatic outcome & & & \\
$\quad$ Improved & $31(39 \%)$ & $67(45 \%)$ & NS \\
$\quad$ Unchanged & $28(35 \%)$ & $51(34 \%)$ & NS \\
$\quad$ Worse & $21(26 \%)$ & $32(21 \%)$ & NS \\
Change in 6 minute walk distance (m) & $+52(14)^{\star \star \star}$ & $+43(8)^{\star \star \star \star}$ & NS \\
Echocardiographic changes & & & \\
$\quad$ LVEDD (mm) & $-1.2(0.8)$ & $-1.8(0.5)^{\star \star \star}$ & NS \\
LVESD (mm) & $-3.3(0.9)^{\star \star \star}$ & $-3.5(0.6)^{\star \star \star \star}$ & NS \\
LVFS (\%) & $+3.3(0.7)^{\star \star \star \star}$ & $+3.0(0.6)^{\star \star \star \star}$ & NS \\
LVEF (\%) & $+5.5(1.2)^{\star \star \star \star}$ & $+5.1(1.0)^{\star \star \star \star}$ & NS \\
\hline
\end{tabular}

NS, non-significant (for between group comparisons); ${ }^{\star \star \star} \mathrm{p}<0.001{ }^{\star \star \star \star \star} \mathrm{p}<0.0001$ compared to baseline (for within group comparisons).

LVEDD, left ventricular end diastolic dimension; LVESD, left ventricular end systolic dimension; LVFS, left ventricular fractional shortening; LVEF, left ventricular ejection fraction.

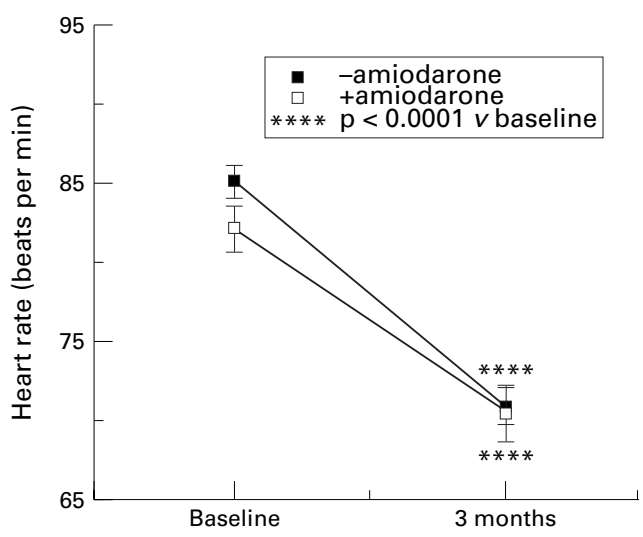

Figure 3 Effect of three months of carvedilol treatment on heart rate stratified according to whether or not the patients were receiving amiodarone at baseline.

higher in this group: $26 \%$ in the amiodarone group versus $25 \%$ in non-amiodarone group (NS). In addition, the need for rehospitalisation for any reason did not differ significantly between the two groups (fig 2).

TOLERABILITY OF CARVEDILOL: HEART RATE The effect of three months' treatment with carvedilol on mean heart rate is shown in fig 3 . Baseline mean resting heart rate before the start of carvedilol treatment was marginally lower in the amiodarone group; however, this difference was not significant $(p=0.11)$. There was a highly significant fall in mean resting heart rate of approximately 15 beats/ min after three months' treatment with carvedilol. The magnitude of the bradycardic response to carvedilol did not differ between the two groups (fig 3). Symptomatic bradycardia necessitating discontinuation of carvedilol occurred in $3 \%$ of patients in both groups.

EFFICACY OF CARVEDILOL

The efficacy of carvedilol, as judged by symptomatic outcome, change in six minute walk distance, and change in echocardiographic left ventricular dimensions and function at three months, is summarised in table 3. The majority of patients reported either symptomatic improvement or were unchanged. Six minute walk distance increased significantly compared to baseline, and left ventricular dimensions decreased significantly. Calculated left ventricular fractional shortening and ejection fraction increased significantly compared to baseline. The differences in symptomatic outcome, six minute walk distance, and echocardiographic variables at three months between the amiodarone and non-amiodarone groups were not significant.

\section{Discussion}

EFFICACY AND TOLERABILITY OF CARVEDILOL The primary findings of this study were that the beneficial effects of carvedilol on left ventricular remodelling, left ventricular systolic function, and symptomatic outcome in patients with heart failure were unaffected by concurrent treatment with amiodarone. Furthermore, carvedilol was well tolerated by these patients. We did observe a trend to more frequent 
adverse events in the amiodarone treated patients; however, this was not associated with a higher rate of permanent discontinuation of carvedilol nor increased need for rehospitalisation. Our findings are consistent with those of Krum and colleagues who conducted a retrospective analysis of the Australian and New Zealand study of carvedilol in patients with heart failure. ${ }^{19}$ They found that the incidence of adverse effects among 19 patients who received carvedilol and amiodarone together was no higher than among patients who received either drug alone.

IMPACT OF AMIODARONE ON ADVERSE EVENTS WITH CARVEDILOL

The increase in adverse events observed in the amiodarone group was of marginal significance and may simply be a chance finding. The incidence of bradycardia necessitating withdrawal of carvedilol was low in our study and consistent with that reported by other investigators. $^{52021}$ Withdrawal of carvedilol because of bradycardia was no higher in patients who were receiving both amiodarone and carvedilol. The major difference in adverse events between the two groups was a higher incidence of worsening heart failure in the amiodarone group. While we cannot exclude an adverse interaction between amiodarone and carvedilol as the basis for this difference, we believe that a more likely explanation is the baseline differences between the two groups. Patients in the amiodarone group were more likely to be in atrial fibrillation and to have a history of ventricular tachycardia. Although the prognostic importance of these arrhythmias in heart failure has been disputed, ${ }^{22-24}$ several recent studies suggest that they may be associated with an increase in morbidity and mortality in patients with heart failure..$^{25-29}$

EFFECT OF CARVEDILOL AND AMIODARONE ON MORTALITY

The impact of combined amiodarone and $\beta$ blockers on mortality in patients with heart failure is not known. Previous studies assessing the effect of amiodarone on survival in patients with heart failure have included few patients who were also taking $\beta$ blockers. ${ }^{11}{ }^{30}$ However, in the two large multicentre studies that examined the effect of amiodarone on survival after myocardial infarction, ${ }^{31}{ }^{32}$ any beneficial effect of amiodarone on cardiac mortality appeared to be confined to those patients who were concurrently treated with $\beta$ blockers. The higher prevalence of prognostically significant arrhythmias at baseline in the amiodarone treated patients in our study suggests that they faced an increased mortality risk compared to the non-amiodarone group. ${ }^{252628} 29$ In spite of this, survival of amiodarone treated patients after initiation of carvedilol was similar to that of patients who had not been treated with amiodarone before receiving carvedilol. This observation needs to be interpreted cautiously but it does suggest that the combination of carvedilol with amiodarone is safe.
EFFECT OF CARVEDILOL ON SYMPTOMATIC OUTCOME AND EXERCISE DURATION

Our findings with regard to the effects of carvedilol on symptomatic outcome and exercise duration suggest a more favourable response to carvedilol with regard to these parameters than has been reported in placebo controlled trials. ${ }^{320}$ The greater benefit observed in our series may be because of a placebo response ${ }^{33}$ or the beneficial effects of regular attendance at a heart failure clinic, ${ }^{34} 35$ rather than a direct effect of carvedilol. Alternatively, the better symptomatic response could be explained in part by the fact that the large majority of patients included in our series were NYHA class III or IV at baseline. There may be an inherent bias towards greater improvements in symptomatic outcome and exercise duration in patients with severe baseline limitations compared to those with less severe symptoms at baseline. ${ }^{21}$ Irrespective of the mechanism of benefit, our findings show that concurrent amiodarone treatment does not alter the beneficial effect of carvedilol on symptomatic outcome or exercise duration.

EFFECT OF CARVEDILOL ON LEFT VENTRICULAR DIMENSIONS AND SYSTOLIC FUNCTION

The reductions in left ventricular diastolic and systolic dimensions and the improvements in left ventricular systolic function in response to carvedilol observed in this series were similar in magnitude to the changes reported by others. ${ }^{134}$ Amiodarone has been shown in several studies to increase left ventricular ejection fraction significantly in patients with heart failure, ${ }^{8-10} 36$ although this has not been a universal finding. ${ }^{37}$ Furthermore, amiodarone's effects on left ventricular remodelling are less clear.

In one study, Trobaugh and colleagues reported that the increase in left ventricular ejection fraction that occurred after an average of two months' treatment with amiodarone was accompanied by a significant increase in left ventricular end diastolic volume and no change in end systolic volume. ${ }^{36}$ The average dose of amiodarone used in that study, however, was considerably higher than the doses used in more recent studies. In another study, Mahmarian and colleagues reported that a daily dose of $100 \mathrm{mg}$ amiodarone for three months significantly increased left ventricular ejection fraction compared to placebo in patients with heart failure. ${ }^{9}$ They also reported that left ventricular end systolic volume decreased, while end diastolic volume was unchanged. Regardless of the effects of amiodarone on left ventricular ejection fraction and remodelling, it is apparent from our study that the beneficial effects of carvedilol on these parameters were unaffected by prior treatment with amiodarone. It is possible that some of our patients had not been fully "loaded" with amiodarone before the start of carvedilol treatment. However, exclusion of patients who had received amiodarone for less than three months from the analysis did not alter the beneficial effects of carvedilol on left ventricular dimensions or systolic function. Our findings indicate that the beneficial effects of carvedilol on left 
ventricular systolic function are independent of and may be additive to those of amiodarone.

STUDY LIMITATIONS

The two major limitations of this study were the non-randomised administration of carvedilol and the lack of a placebo control group. The former probably accounts for the baseline differences observed between the amiodarone and the non-amiodarone groups. The lack of a placebo group may have resulted in an overestimate of the beneficial effects of carvedilol on symptomatic outcome and exercise duration. However, we consider that a placebo response is unlikely to account for the beneficial effects of carvedilol on left ventricular dimensions and systolic function that we observed, particularly as the magnitude of the benefits in these parameters was highly consistent with that reported in placebo controlled studies.

CONCLUSIONS

The beneficial effects of carvedilol on left ventricular remodelling, systolic function, and symptomatic status are not affected by concurrent treatment with amiodarone. Adverse events during treatment with carvedilol tend to occur more frequently in patients who are also receiving amiodarone. However, the need for rehospitalisation or permanent withdrawal of carvedilol is not increased. Concurrent treatment with amiodarone should not be considered a contraindication to the use of carvedilol in heart failure.

Carvedilol was kindly supplied by SmithKline Beecham International, a division of SmithKline Beecham (Australia) Pty Ltd.

1 Doughty R, Whalley G, Gamble G, et al and the Australia and New Zealand Heart Failure Collaborative Research Group. Left ventricular remodelling with carvedilol in patients with congestive heart failure due to ischaemic heart disease. $\mathcal{F}$ Am Coll Cardiol 1997;29:1060-6.

2 Metra M, Nardi M, Giubbini R, et al. Effects of short- and long-term carvedilol administration on rest and exercise hemodynamic variables, exercise capacity and clinical conditions in patients with idiopathic dilated cardiomyopathy. ditions in patients with idiopathic dilat

3 Olsen S, Gilbert E, Renlund D, et al. Carvedilol improves left ventricular function and symptoms in chronic heart failure: a double-blind randomized study. $\mathcal{F}$ Am Coll Cardio 1995;25:1225-31.

4 Bristow M, Gilbert E, Abraham W, et al. Carvedilol produces dose-related improvements in left ventricular function and survival in subjects with chronic heart failure. Circulation 1996;94:690-7.

5 Packer M, Bristow M, Cohn J, et al. The effect of carvedilol on morbidity and mortality in patients with chronic heart failure. N Engl F Med 1996;334:1349-55.

6 Colucci W, Packer M, Bristow M, et al. Carvedilol inhibits clinical progression in patients with mild symptoms of heart failure. Circulation 1996;94:2800-6.

7 Australia and New Zealand Heart Failure Collaborative Research Group. Randomised, placebo-controlled trial of carvedilol in patients with congestive heart failure due to carvedilol in patients with congestive heart failure
ischaemic heart disease. Lancet 1997;349:375-80.

8 Hamer AW, Arkles LB, Johns JA. Beneficial effects of low dose amiodarone in patients with congestive cardiac failure: a placebo-controlled trial [see comments]. $\mathcal{f} \mathrm{Am}$ failure: a placebo-controlled

9 Mahmarian JJ, Smart FW, Moye LA, et al. Exploring the minimal dose of amiodarone with antiarrhythmic and hemodynamic activity. Am F Cardiol 1994;74:681-6.

10 Massie BM, Fisher SG, Deedwania PC, et al. Effect of amiodarone on clinical status and left ventricular function in patients with congestive heart failure: CHF-STAT investigators. Circulation 1996;93:2128-34.

11 Doval HC, Nul DR, Grancelli HO, et al. Randomised trial of low-dose amiodarone in severe congestive heart failure: grupo de estudio de la sobrevida en la insuficiencia cardiaca en Argentina (GESICA) [see comments]. Lancet 1994;344:493-8.

12 Amiodarone Trials Meta-Analysis Investigators. Effect of prophylactic amiodarone on mortality after acute myocarprophylactic amiodarone on mortality after acute myocar-
dial infarction and in congestive heart failure: meta-analysis of individual data from 6500 patients in randomised trials. Lancet 1997;350:1417-24.
13 Singh BN, Venkatesh N, Nademanee K, et al. The historical development, cellular electrophysiology and pharmacology
of amiodarone. Prog Cardiovasc Dis 1989;31:249-80.

14 Freedman MD, Somberg JC. Pharmacology and pharmacokinetics of amiodarone. $\mathcal{F}$ Clin Pharmacol 1991;31:1061-9.

15 Drvota V, Haggblad J, Blange I, et al. The effect of amiodarone on the beta-adrenergic receptor is due to a downregulation of receptor protein and not to a receptor-ligand interaction. Biochem Biophys Res Commun 1999;255:515-20.

16 Haikerwal D, Dart AM, Little PJ, et al. Identification of a novel, inhibitory action of amiodarone on vesicular monoan

17 Stevenson WG, Stevenson LW, Middlekauff HR, et al. Improving survival for patients with advanced heart failure: a study of 737 consecutive patients. $7 \mathrm{Am}$ Coll Cardiol a study of 737 con

18 Teichholz L, Kreulen T, Herman M, et al. Problems in echocardiographic volume determinations: echocardiographic-angiographic correlations in the presence and absence of asynergy. Am f Cardiol 1976;37:7-11.

19 Krum H, Shusterman N, MacMahon S, et al. Efficacy and safety of carvedilol in patients with chronic heart failure receiving concomitant amiodarone therapy: Australia and New Zealand heart failure research collaborative group. fournal of Cardiac Failure 1998;4:281-8.

20 Australia and New Zealand Heart Failure Collaborative Research Group. Effects of carvedilol, a vasodilator beta-blocker, in patients with congestive heart failure due to ischaemic heart disease. Circulation 1995;92:212-18.

21 Krum H, Sackner-Bernstein J, Goldsmith R, et al. Doubleblind, placebo-controlled study of the long-term efficacy of carvedilol in patients with severe chronic heart failure. Circulation 1995;92:1499-506.

22 Carson PE, Johnson GR, Dunkman WB, et al. The influence of atrial fibrillation on prognosis in mild to moderate heart failure: the V-HeFT studies. Circulation 1993;87(suppl VI):VI-102-10.

23 Stevenson WG, Stevenson LW, Middlekauff HR, et al. Improving survival for patients with atrial fibrillation and advanced heart failure [published erratum appears in $\mathcal{f} \mathrm{Am}$ advanced heart failure [published erratum appears in 7 Am 1458-63.

24 Packer M. Lack of relation between ventricular arrhythmias and sudden death in patients with chronic heart failure. Circulation 1992;85(suppl I):I-50-6.

25 Benjamin EJ, Wolf PA, D'Agostino RB, et al. Impact of atrial fibrillation on the risk of death: the Framingham heart study. Circulation 1998;98:946-52.

26 Dries DL, Exner DV, Gersh BJ, et al. Atrial fibrillation is associated with an increased risk for mortality and heart failure progression in patients with asymptomatic and symptomatic left ventricular systolic dysfunction: a retrospective analysis of the SOLVD trials. F Am Coll Cardiol 1998;32:695-703.

27 Pozzoli M, Cioffi G, Traversi E, et al. Predictors of primary atrial fibrillation and concomitant clinical and hemodynamic changes in patients with chronic heart failure: a prospective study in 344 patients with baseline sinus rhythm. F Am Coll Cardiol 1998;32:197-204.

28 Podrid PJ, Fogel RI, Fuchs TT. Ventricular arrhythmia in congestive heart failure. Am f Cardiol 1992;69:82G-95G; discussion 95G-96G.

29 Doval HC, Nul DR, Grancelli HO, et al. Nonsustained ventricular tachycardia in severe heart failure: independent marker of increased mortality due to sudden death. GESICA- GEMA investigators [see comments]. Circulation 1996;94:3198-203.

30 Singh SN, Fletcher RD, Fisher SG, et al. Amiodarone in patients with congestive heart failure and asymptomatic entricular arrhythmia: survival trial of antiarth therapy in congestive heart failure [see comments]. $N$ Engl f Med 1995;333:77-82

31 Cairns JA, Connolly SJ, Roberts R, et al. Randomised trial of outcome after myocardial infarction in patients with frequent or repetitive ventricular premature depolarisations: Canadian amiodarone myocardial infarction arrhythmia trial investigators (CAMIAT) [published erratum appears in Lancet 1997;349:1776] [see comments]. Lancet 1997;349:675-82.

32 Julian DG, Camm AJ, Frangin G, et al. Randomised trial of effect of amiodarone on mortality in patients with left-ventricular dysfunction after recent myocardial infarction: European myocardial infarct amiodarone trial investigators (EMIAT) [published errata appear in Lancet investigators (EMIAT) [published errata appear in Lancet 1997;349:1180 and $1997 ; 349: 667-74$

33 Packer M. The placebo effect in heart failure. Am Heart $\mathcal{F}$ 1990;120:1579-82.

34 Rich MW, Beckham V, Wittenberg C, et al. A multidisciplinary intervention to prevent the readmission of elderly patients with congestive heart

35 Smith LE, Fabbri SA, Pai R, et al. Symptomatic improvement and reduced hospitalization for patients attending cardiomyopathy clinic. Clin Cardiol 1997;20:949-54.

36 Trobaugh GB, Kudenchuk PJ, Greene HL, et al. Effect of amiodarone on ventricular function as measured by gated radionuclide angiography. Am f Cardiol 1984;54:1263-6.

37 Cleland JG, Dargie HJ, Findlay IN, et al. Clinical, haemodynamic, and antiarrhythmic effects of long term treatment with amiodarone of patients in heart failure. Br Heart $\mathcal{F}$ 1987;57:436-45. 